\title{
Invited Article: Digital beam-forming imaging riometer systems
}

\author{
Farideh Honary, ${ }^{1, \text { a) }}$ Steve R. Marple, ${ }^{1}$ Keith Barratt, ${ }^{2}$ Peter Chapman, ${ }^{1}$ \\ Martin Grill, ${ }^{3}$ and Erling Nielsen ${ }^{4}$ \\ ${ }^{1}$ Department of Physics, Lancaster University, Lancaster LA1 4YB, United Kingdom \\ ${ }^{2}$ Cable Sense Ltd., Lancaster LA1 4XQ, United Kingdom \\ ${ }^{3}$ SRI International, Menlo Park, California 94025, USA \\ ${ }^{4}$ Max Planck Institute for Solar System Research, 37191 Katlenburg-Lindau, Germany
}

(Received 18 November 2010; accepted 14 February 2011; published online 22 March 2011)

\begin{abstract}
The design and operation of a new generation of digital imaging riometer systems developed by Lancaster University are presented. In the heart of the digital imaging riometer is a field-programmable gate array (FPGA), which is used for the digital signal processing and digital beam forming, completely replacing the analog Butler matrices which have been used in previous designs. The reconfigurable nature of the FPGA has been exploited to produce tools for remote system testing and diagnosis which have proven extremely useful for operation in remote locations such as the Arctic and Antarctic. Different FPGA programs enable different instrument configurations, including a $4 \times 4$ antenna filled array (producing $4 \times 4$ beams), an $8 \times 8$ antenna filled array (producing 7 $\times 7$ beams), and a Mills cross system utilizing 63 antennas producing 556 usable beams. The concept of using a Mills cross antenna array for riometry has been successfully demonstrated for the first time. The digital beam forming has been validated by comparing the received signal power from cosmic radio sources with results predicted from the theoretical beam radiation pattern. The performances of four digital imaging riometer systems are compared against each other and a traditional imaging riometer utilizing analog Butler matrices. The comparison shows that digital imaging riometer systems, with independent receivers for each antenna, can obtain much better measurement precision for filled arrays or much higher spatial resolution for the Mills cross configuration when compared to existing imaging riometer systems. () 2011 American Institute of Physics. [doi:10.1063/1.3567309]
\end{abstract}

\section{INTRODUCTION}

A riometer (relative ionospheric opacity meter ${ }^{1}$ ) measures to what extent the cosmic background noise is absorbed by the ionosphere. Riometers are used, often in conjunction with other instruments, to study ionospheric processes which in turn relate to space weather and high frequency (HF) communications links. Most of the ionospheric absorption occurs in the $\mathrm{D}$ region of the ionosphere, primarily due to high energy particle precipitation (electrons and protons) and ionization by UV and $x$ rays. For riometry purposes the average height of the absorbing layer is usually considered to be 90 $\mathrm{km}$. Riometers are typically classified as either wide beam or imaging; both are now introduced in more detail.

Wide-beam riometers consist of a simple antenna system with an azimuthally symmetric radiation pattern; consequently, they have a wide field of view but no imaging capabilities. The antenna is connected to a receiving device which measures the power of the incoming "signal" (cosmic background noise). In the absence of geomagnetic activity the received power forms a baseline, or "quiet day curve" from which absorption measurements can be derived. Due to their relatively simple construction, wide-beam riometers are inexpensive to build and operate. They are useful for a general overview of the current state of the ionosphere,

a) Electronic mail: f.honary@ lancaster.ac.uk. but do not provide any information on the spatial structure and dynamics within their field of view. There are over 50 wide-beam riometers in operation worldwide.

Using a narrow-beam antenna Nielsen and Axford ${ }^{2}$ demonstrated that the high-energy electron precipitation had spatial and dynamic structures similar to those observed optically for lower energies in association with the aurora. Kikuchi, Yamagishi, and $\mathrm{Sato}^{3}$ used a scanning riometer system $^{4}$ to observe cosmic noise absorption pulsations with a large azimuthal $m$-number that wide-beam riometer chains would have difficulty in observing. However, the scanning riometer could only observe a cross-shaped area of the sky. The need for higher spatial resolution and simultaneous observation of the viewing area led to the development of imaging riometers. There are currently more than 25 imaging riometers in operation globally, working at frequencies between 30 and $40 \mathrm{MHz}$.

The first imaging riometer, known as IRIS (imaging riometer for ionospheric studies), was deployed at the South Pole in $1998 .{ }^{5}$ Utilizing $8 \times 8=64$ crossed-dipoles configured as a filled phased array ${ }^{6,7}$ and a modified twodimensional (2D) analog Butler matrix, ${ }^{8}$ it produces $7 \times 7$ $=49$ imaging beams. Beam width at the zenith is $12.8^{\circ}$, which translates to $20.2 \mathrm{~km}$ at a height of $90 \mathrm{~km}$. To simplify the hardware requirements IRIS scans each row once every second; thus, only 7 riometers (one per column) are required instead of 49 (one per beam). As a result of the scanning process each beam is switched to a riometer for $95 \mathrm{~ms}$, and the noise-balancing technique employed means 
that the antenna signal is observed for just $47.5 \mathrm{~ms}$. Further development led to a smaller $4 \times 4$ antenna system with 16 beams for use in automated geophysical observatories (PENGUIN IRIS); ${ }^{9}$ the low power requirements also necessitated a reduction in cadence to $12 \mathrm{~s}$. By cadence we mean the time interval between the start of successive measurements, as defined by the Space Physics Archive Search and Extract (SPASE) data dictionary. ${ }^{10}$ Based on the successful IRIS design other groups around the world constructed similar instruments, such as an $8 \times 8$ system using an unmodified Butler matrix to create 64 beams, ${ }^{11}$ deployed at Tjornes, Iceland. While the unmodified Butler matrix results in an enlarged field of view, modeling shows that it comes at the cost of increased side lobes in the outer and corner beams. The system at Tjornes also differs in that the antenna spacing has been increased to $0.65 \lambda$, which reduces the field of view to increase angular resolution.

The largest phased array imaging riometer is located at Poker Flat, Alaska. ${ }^{12}$ It utilizes $16 \times 16=256$ antennas and standard Butler matrices to produce 256 beams with a maximum cadence of $1 \mathrm{~s}$. It achieves a beam width of $6.6^{\circ}$ at the zenith. However, for 48 phasing patterns with lobes at larger zenith angles there is no production of a clear main lobe; thus, the number of usable beams is 208 . To increase the spatial resolution of a filled phased array antenna by $n$, the number of required antenna elements increases with $n^{2}$, rendering very high resolution phased array antennas impractical. A $16 \times 16$ antenna array presents a significant expense, which is one reason why only one such system exists. The physical requirements are substantial, a 256 antenna array operating at $38.2 \mathrm{MHz}$ requires a flat area of over $60 \times 60 \mathrm{~m}$ due to the antenna spacing dimensions at this frequency. ${ }^{12}$

The measurement precision limit at present is around 0.04-0.05 dB at $1 \mathrm{~s}$ cadence for an $8 \times 8$ antenna filled array system. ${ }^{5,13}$ The need for yet higher spatial resolution to resolve small-scale structure, for example, in studies utilizing riometer and optical data, ${ }^{14,15}$ and riometer and radar data, ${ }^{16}$ plus the requirement for better measurement precision, for example, to determine the quiet absorption level, ${ }^{17}$ has led to a new design of digital imaging riometers. In this paper we show how digital beam forming provides a range of configurable options to fulfill the requirements of higher spatial resolution, greater measurement precision, and high time resolution. We describe three different imaging riometer implementations in their chronological order of development. First, the Advanced Rio-Imaging Experiment in Scandinavia (ARIES) is based on the Mills cross design with $32+32=64$ antennas. In our implementation there are 63 antennas; the 15th antenna in each arm being common to both arms. However, this is not a necessary condition so we will use the notation $32+32=64$ for a Mills cross system with two arms of 32 antennas each. We also describe two designs of filled phased array imaging riometers, one using an $8 \times 8=64$ antenna array and the other with a $4 \times 4=16$ antenna array. All systems use the same modular hardware and we report the performance improvements obtained by each system.

\section{MILLS CROSS SYSTEM, WITH 32 + 32 ANTENNAS}

The Mills cross technique ${ }^{18-20}$ provides a means to fulfill the requirement of higher spatial resolution. Such a riometer is based on the principle of cross-correlating signals from the two perpendicular fan beams formed by the perpendicular linear phased arrays, as first conceived and used by Mills ${ }^{21}$ and Christiansen and Mathewson ${ }^{22}$ albeit not for riometry purposes. The main advantage of this type of antenna arrangement is that it can achieve high spatial resolution with significantly fewer antenna elements when compared to a filled array antenna. ${ }^{23}$ To achieve $n$ times the resolution, the number of antenna elements required now only increases linearly with $n$ and not $n^{2}$. A three-dimensional illustration of the beam-forming process for a Mills cross antenna array is shown in Fig. 1. The process can be broken down into two discrete stages:

Stage One-Fan beams. Initially, each arm of the cross is considered as a separate linear phased array. Each arm forms several "fan beams," the pointing directions of which are determined by a one-dimensional (1D) digital beam-forming matrix. By themselves, the fan beams still cover a large solid angle, the spatial resolution in one direction is still very poor.

Stage Two-Pencil beams. The concept that turns the Mills cross antenna array into a high resolution array is to cross-correlate the signals from two perpendicular fan beams. One can consider the cosmic background as many independent noise sources distributed over the visible hemisphere. The cross-correlation process extracts the signals that originate from the overlapping region of the two fan beams (our "signal"). Noise sources which are within the two fan beams but outside of the common area degrade the signal-to-noise
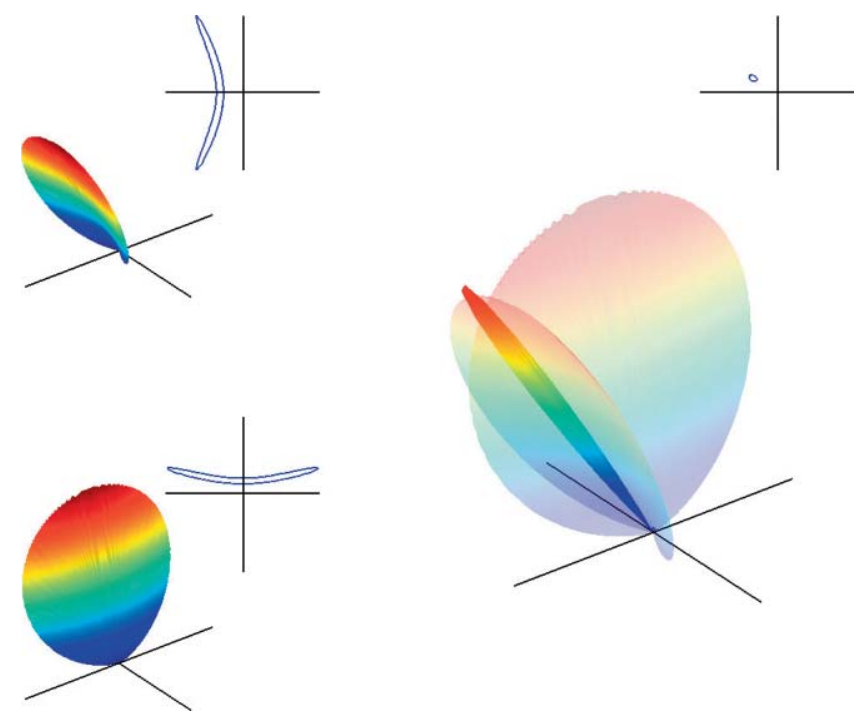

FIG. 1. (Color) Beam forming for a Mills cross imaging riometer system. The two small panels on the left show an example of a fan beam formed by a linear array of antennas along the $x$-axis (top panel) and along the $y$-axis (bottom panel), respectively. The small inset in the upper right-hand corner of each panel shows the $-3 \mathrm{~dB}$ outline of the fan beam in plan view. The large panel on the right-hand side depicts the cross-correlation process. Two perpendicular fan beams (shaded) are cross-correlated to produce a narrow pencil beam (solid) pointing in the direction where the two fan beams intersect. The inset shows the $-3 \mathrm{~dB}$ contour of the pencil beam at the same scale as the other inset diagrams. 


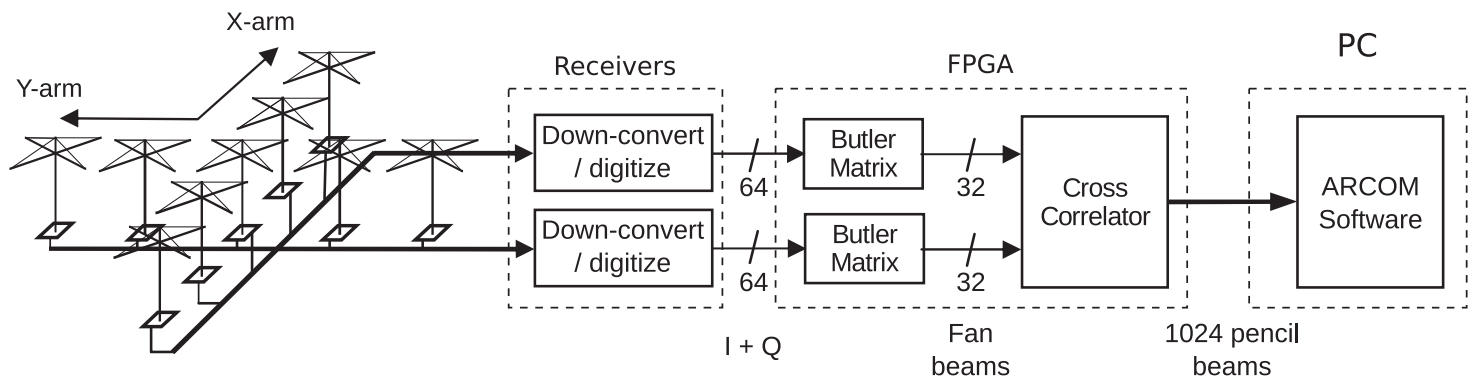

FIG. 2. Mills cross block diagram. The total number of antennas actually used is 63, 32 in each arm with one antenna common to both arms. (ARCOM denotes Advanced Riometer Components software.)

ratio (SNR). This illustration forms the basis of the model by Nielsen, Honary, and Grill. ${ }^{23}$ A narrow pencil beam is therefore formed for each combination of fan beam from one arm with a fan beam from the other arm of the Mills cross.

A drawback of a Mills cross-type system, compared to a filled phased array system of similar resolution, is that the achievable noise performance is worse due to the reduced aperture of the antenna array. This issue has been investigated in Nielsen, Honary, and Grill ${ }^{23}$ and Hagfors, Grill, and Honary ${ }^{24}$ showing that it is theoretically feasible to successfully implement a riometer system based on the Mills cross technique. To achieve this, the bandwidth-time product must be maximized by increasing the receiver bandwidth and the proportion of each second for which data is integrated.

Based on the Mills cross design we have devised a working imaging riometer system, the Advanced Rio-Imaging Experiment in Scandinavia (ARIES), an overview of which is given in Fig. 2. The system can be separated into three main parts, antennas and receivers, digital beam forming, and data acquisition and processing.

\section{A. Antennas and receivers}

The ARIES antenna array consists of two arms of 32 antennas aligned in geographic North-South and EastWest directions. Each antenna is a freestanding half-wave crossed dipole ${ }^{25}$ with an azimuthally symmetric radiation pattern and spaced at $\lambda / 2$ from its neighbors. The dipoles are aligned NE-SW and NW-SE. There are 63 antennas in total, the central one is shared between the two arms. The crossed dipoles are circularly polarized to match the polarization of the more strongly absorbed X-mode incoming "signal" from the cosmic noise background. Each antenna is mounted on a wooden mast at a height of $1.96 \mathrm{~m}$, i.e., $\lambda / 4$ for the center frequency of $38.235 \mathrm{MHz}$.
The mast and each of the four antenna elements are supported by guy ropes. A ground plane one wavelength wide, made of metal mesh, is laid out under both arms of the array. Lowloss coaxial cables of electrically identical length (to within $\pm 1^{\circ}$ phase accuracy at $38.235 \mathrm{MHz}$ ) connect each antenna to the receiver hardware in the hut. The signal from the common antenna is fed into one receiver whose output is digitally duplicated for both the $\mathrm{X}$ and $\mathrm{Y}$ arms.

A low-noise multichannel simultaneously sampled receiver has been developed specifically for use in digital riometry. A modular approach was taken to allow for ease of construction, development, testing, and on-site servicing. The receiver channels are paired so that two receiver channels are colocated on a standard $220 \times 100 \mathrm{~mm}$ Eurocard printed circuit board. The block diagram of a single homodyne channel is shown in Fig. 3. Each channel has two inputs to allow for the receiver to be switched to a calibration signal, which is important for the accurate power calculations required. The control lines for all solid-state RF switches are connected together and are controlled by the data recording computer. The filtered RF signal is amplified by a Minicircuits MAR-8ASM monolithic microwave integrated circuit amplifier and split into two separate signals. One is mixed with the local oscillator (LO) signal and the other with a $90^{\circ}$ phase-shifted version of the LO signal to form in-phase (I) and quadrature (Q) signals.

The diplexer provides the mixer with $50 \Omega$ termination over a wide frequency range. This ensures the intermodulation and port isolation performance of the mixer is not degraded. After down-conversion the baseband signals undergo three cascaded amplifications and filter stages before entering a simultaneously sampling 14 bit analog to digital converter (ADC). The gain of the second amplification stage can be programmed from the field-programmable gate array (FPGA) us-

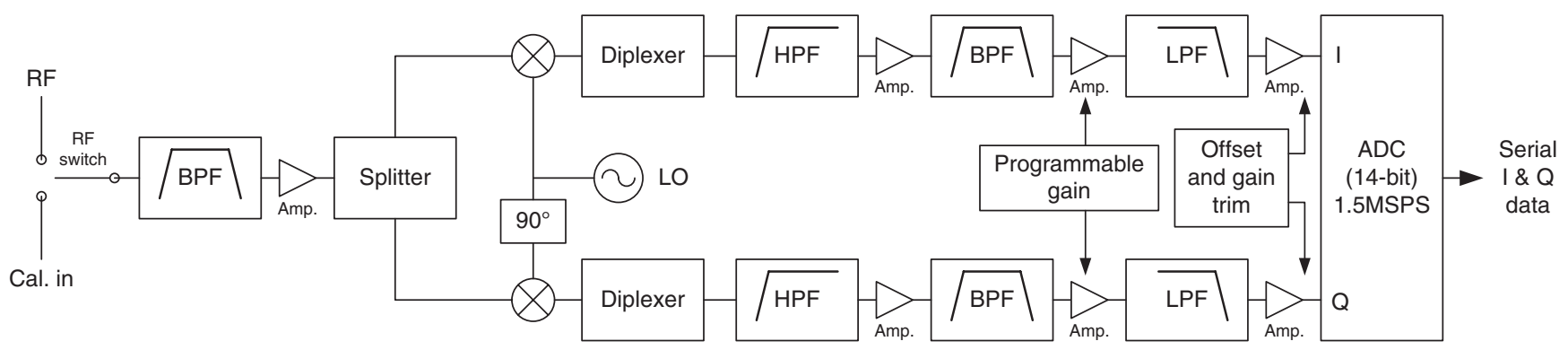

FIG. 3. Receiver block diagram. The diplexer provides the mixer with $50 \Omega$ termination to ensure that the intermodulation and port isolation performance is not degraded. (BPF denotes band-pass filter; HPF denotes high-pass filter; LPF denotes low-pass filter.) 
ing a 3 bit digital control; gain controls for all receiver modules are ganged together. At the third amplification stage the gain of the I and Q signals can be trimmed to enable a precise match, and dc offsets can be removed separately.

To obtain the required measurement precision the bandwidth-time product was maximized by setting the receiver bandwidth to $1 \mathrm{MHz}$ as opposed to the typical $200 \mathrm{kHz}$ used by analog beam-forming imaging riometers; an earlier site survey had found no man-made sources of noise in the band 37.7-38.7 MHz. Additionally, by employing independent receivers, data from each beam are integrated for more than $95 \%$ of each second [the remaining $5 \%$ is used by the FPGA for transferring the data to the host personal computer (PC)] as opposed to less than $5 \%$ for typical IRIS-type riometers, which multiplex one receiver between seven beams and switch between received power and calibration noise source with a duty cycle of $50 \%$ (noise-balancing technique). ${ }^{5}$ The receiver bandwidth requirement of $1 \mathrm{MHz}$ was met by the ADC sampling at $1.47 \mathrm{MHz}$. Each ADC outputs multiplexed in-phase and quadrature serial data onto a single data line, thus reducing the wiring between receiver rack and PCmounted signal processing card. The inputs to each receiver board are the local oscillator signal, the control signals for the programmable gain amplifier, the digital clock, and the convert signal for the ADC.

All receiver boards required for the complete system are housed along with the local oscillator, digital clock generator, calibration noise source, and other peripheral electronics in an industry-standard $12 \mathrm{U} 19^{\prime \prime}$ subrack. The three supply voltages $(12 \mathrm{~V},-5 \mathrm{~V}$, and $+5 \mathrm{~V})$ required for the receiver electronics are provided by a set of linear power supplies. These are also modular in construction and are housed in an additional $19^{\prime \prime}$ subrack.

The digital clock signal is generated by a commercial temperature-compensated crystal oscillator module operating at $50 \mathrm{MHz}$. The digital clock is divided by a factor of 34 to produce the ADC convert signal at $1.470588 \mathrm{MHz}$. The local oscillator signal is generated by a Colpitts oscillator with the crystal operating as a filter; the entire LO circuitry is housed within a temperature-controlled oven. To prevent harmonics of the convert signal affecting the receiver, the local oscillator frequency was set to $38.235294 \mathrm{MHz}$; thus, the 26th harmonic of the ADC convert signal exactly matches the local oscillator frequency; any harmonics from the convert signal which enter the receiver either fall outside the pass band or are mixed to a dc signal which is blocked by the ac coupling between amplifier stages. The calibration signal source is derived from a noise diode and outputs white noise over the receiver pass band; both the noise diode and distribution amplifier operate inside a miniature temperature-controlled oven. The local oscillator and calibration signals are buffered and distributed to all receivers via power-splitters and phase-matched coaxial cables.

\section{B. Digital beam forming}

Previous imaging riometer designs (e.g. Detrick and Rosenberg, ${ }^{5}$ Murayama et al.,${ }^{12}$ and Yamagishi et al. ${ }^{11}$ ) have been constructed using analog Butler matrices. Technological advances have enabled us to implement one-dimensional digital Butler matrices using an FPGA to evaluate a fast Fourier transform (FFT), which is the digital equivalent of the analog Butler matrix. ${ }^{6}$ The digital approach provides a number of advantages: size and cost are greatly reduced, programmable tapering of the array is readily implemented, reduced losses and variability due to the reduction in analogue electronics, and better real-time diagnostic capability of individual antennas and receiver channels. However, the computational requirements are demanding; the receivers output data at a rate of $2.63 \mathrm{Gbit} / \mathrm{s}(64$ receivers $\times 2$ complex channels $\times 14$ bits/sample $\times 1.47$ Msamples/s). In addition, beam forming for a Mills cross requires the cross-correlation of data from all sets of perpendicular fan beams in real time. Through appropriate parallelization it was possible to implement both beam-forming stages and the cross-correlator onto a single Xilinx Virtex-II ${ }^{\mathrm{TM}} \mathrm{XC} 2 \mathrm{~V} 8000$ field-programmable gate array.

Although the beam-forming process creates 1024 beams not all of these are deemed usable; for some the fans beams do not fully intersect (i.e., their $-3 \mathrm{~dB}$ gain contours do not intersect), while others with large zenithal angles $\left(>55^{\circ}\right)$ have larger side lobes than is desirable. Although data from all 1024 beams are recorded the postprocessing software normally uses data from the 556 beams with zenith angles $<55^{\circ}$ and for which the pair of perpendicular fan beams fully intersect. A Mills cross riometer comprising two arms of 32 antennas each can theoretically achieve a beam width at the zenith of $4.5^{\circ}$, although with higher side lobes $(-6.5 \mathrm{~dB})$ than for a filled array. ARIES utilizes cosine tapering to reduce the side lobes, which range from $-17.4 \mathrm{~dB}$ at the center of the field of view to $-13.1 \mathrm{~dB}$ for beams with zenith angles of $54.9^{\circ}$; this is actually better than for IRIS riometers, which range from $-13.3 \mathrm{~dB}$ for the zenithal beam to $-5.37 \mathrm{~dB}$ for the corner beams (or $-9.09 \mathrm{~dB}$ for the beams adjacent to the corner beams). As a result of tapering the beam width at the zenith is enlarged to $6.56^{\circ}$. This beam width is achieved with 63 antennas; for comparable performance from a filled-array 256 antennas would be required.

A block diagram of the FPGA processing software is shown in Fig. 4. The 32 in-phase and quadrature outputs from the X-arm are double-buffered and then converted to one serial data stream. To remove any offsets from the ADCs the signals are then passed through a first-order high-pass digital filter with a corner frequency of $9.7 \mathrm{~Hz}$. The dc offset removal is applied independently to each of the 64 serial data values. Each receiver output can be tapered and/or rotated by multiplying the in-phase/quadrature pair with a complex tapering coefficient (real and imaginary parts are each signed 16 bit values). The 32 receiver signals are passed through a one-dimensional FFT which outputs 32 complex signals (analogous to 32 fan beam outputs) to the cross-correlator. An identical parallel processing stream operates on data from the Y-arm. From its 64 complex inputs the cross-correlator generates 1024 complex signals, each with a data rate of 1.47 Msamples/s. All 1024 complex signals are accumulated and output as 64 bit values at 1 s cadence. The FPGA also computes and outputs the signal power from each receiver, again at $1 \mathrm{~s}$ cadence (analogous to 63 independent wide-beam riometers). 


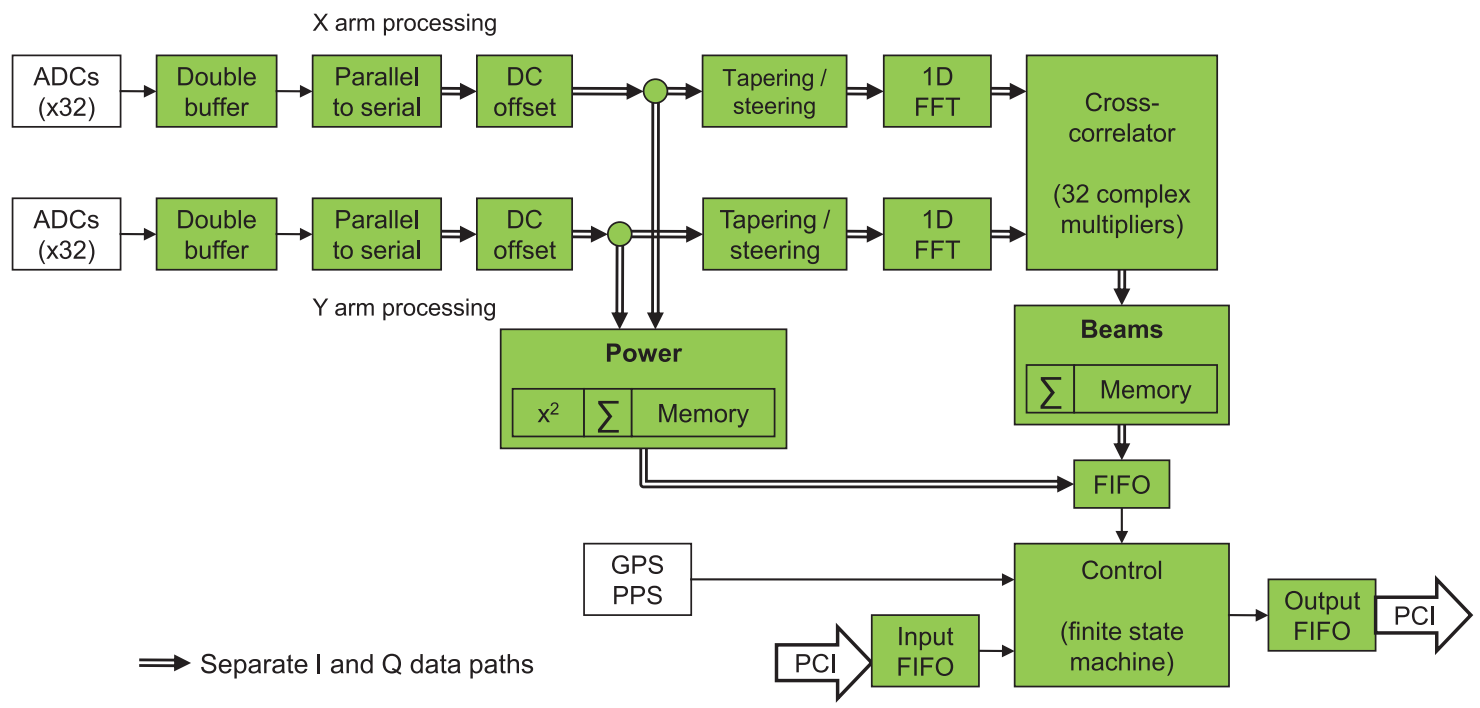

FIG. 4. (Color) Block diagram of the FPGA processing for the ARIES Mills cross. Filled boxes show stages processed within the FPGA. (ARIES denotes Advanced Rio-Imaging Experiment in Scandinavia. FIFO denotes a first in, first out buffer.)

The entire processing system is controlled by a finitestate machine, which is synchronized to the start of the second boundary by a pulse-per-second (PPS) signal from an external global positioning system (GPS) receiver. Periodically, the finite-state machine switches the receiver RF inputs to the calibration noise source to enable autonomous calibration by the postprocessing software. The default behavior can be modified by uploading a parameter block containing various configuration parameters, including the tapering coefficients, on/off durations for the calibration interval timers, and the number of samples to integrate per second. The block diagram shows the latest revision of the FPGA processing software. Originally, the filters for dc offset removal were not included as it was thought that the circuitry contained in the receivers would provide the necessary functionality. However, it was found that temperature variations experienced in the field caused changes in the dc offsets of the ADC; the cross-correlation is very sensitive to such common-mode changes which manifest as an apparent increase in power for the four near-zenithal beams. The introduction of digital filtering overcame this problem. Calculation and recording of receiver power were added to provide wide-beam riometer data.

Additional FPGA programs provide support for system testing and maintenance operations, such as adjustment of the receiver offset and gain controls and inspection of the raw data samples. Execution of the test programs requires the standard recording software to be stopped and the test program to be uploaded to the FPGA, which then reprograms its internal logic gates.

\section{Data acquisition and processing}

ARIES operation is controlled and monitored by an industry-standard PC that connects to the beam-forming FPGA through the industry-standard Peripheral Component Interconnect (PCI) bus. Bespoke Advanced Riometer Components (ARCOM) software ${ }^{26}$ running on the PC is responsible for encapsulating the raw data into an appropriate streaming data format similar to the format used in digital video broadcasting. Data can be disseminated to remote file servers both in real time and in nightly bursts as well as stored locally. Additional processing, for example, postintegration, and visualization components can be plugged in and out as required and without interrupting system operation. All components can be controlled and monitored remotely. The archived raw data stream from ARIES during standard operation amounts to $1.5 \mathrm{~GB} /$ day. Offline data processing, creation of quiet day curves, etc., are performed using the Multi-Instrument Analysis (MIA) package ${ }^{27}$ for MATLAB.

One factor which can affect imaging riometer data quality is scintillation from point sources, in particular the cosmic radio sources Cassiopeia $\mathrm{A}$ and Cygnus $\mathrm{A}$ in the northern hemisphere and Sagittarius A* in the southern hemisphere. Scintillation is caused by ionospheric irregularities and produces rapid fluctuations in the apparent power level, thereby making absorption measurements on short time scales inaccurate. With knowledge of the beam radiation pattern and location of the radio sources, it is possible to predict which beams may be affected and when. Based upon this approach a filter in MIA is able to remove or smooth data which may be affected by scintillation. The level at which this filter is activated can be adjusted, with the threshold given in decibels relative to gain in the beam-pointing direction. The occurrence and number of beams potentially affected by scintillation for various radio sources are given in Table I.

Another factor which can affect data quality in a Mills cross system derives from the correlation process; the antenna gain is complex, which creates both "positive side lobes" and "negative side lobes." ${ }^{24,28}$ When strong signals enter the negative side lobes an apparent decrease in signal power can result. If a negative side lobe coincides with a region of ionospheric absorption the decrease in power is reduced; thus, an apparent increase in signal power is seen. Traditional algorithms for generating the quiet day curve, which often look for the upper received power envelope, must be used with caution. 


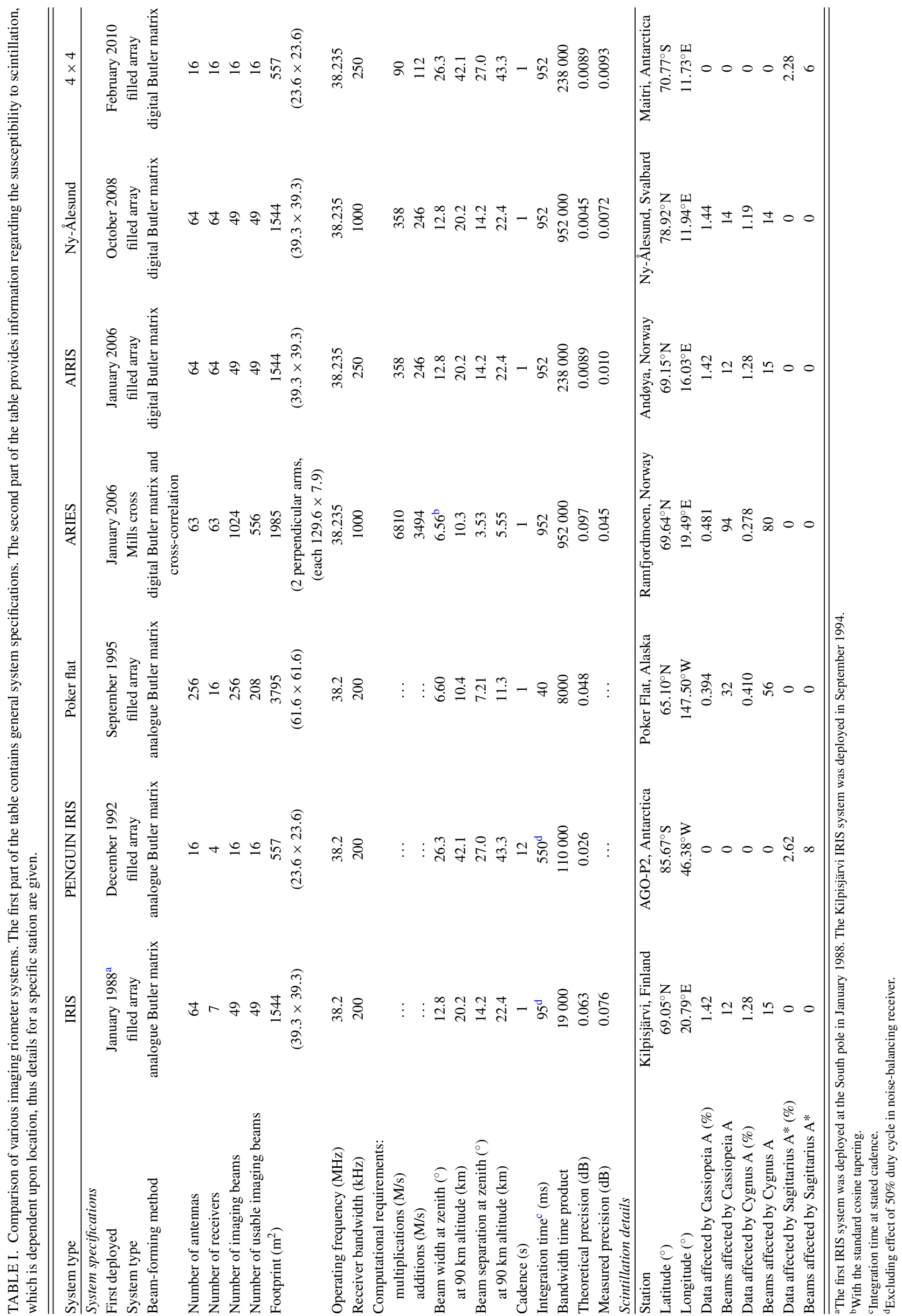


The variation on received power by sources passing through side lobes is not unique to Mills cross riometers, although filled arrays only feature positive side lobes, and illustrates why the control of side lobes is so important. As mentioned previously (Sec. II B) the side lobes in ARIES are better controlled than side lobes of filled array riometers such as IRIS. As a result of the weak side lobes the negative side lobe effect is limited to when the strongest sources, Cassiopeia A and Cygnus A, pass through the negative side lobes, it is not observed at any other time. Thus potentially affected periods can be predicted based upon beam and sidereal time. MIA contains a filter which can remove samples potentially affected by the negative side lobes, similar to the method employed for removing data affected by scintillation.

Based on the theoretical beam projection the occurrence of the radio sources Cassiopeia A and Cygnus A passing through the negative side lobes has been analyzed. It was found that, for ARIES, the loci of Cassiopeia A and/or Cygnus A pass through a negative side lobe for 448 of the 556 beams. The minimum number of beams affected at any one time is zero, the maximum 31 , the mean 12 , and the percentage of total samples affected is just $2.12 \%$. The presence of negative side lobes is more complicated than considering just the loci of radio sources as the effect is also dependent on the strength of the signal received in the main lobe. In practice, the effect is not often noticed but as the possibility of it occurring is predictable, potentially affected portions of the data can be automatically blanked out if required. Filled array imaging riometers do not use a correlation process, their antenna gain is not complex, and the issue of negative side lobes does not arise.

\section{Operation and results}

The ARIES riometer system began collecting data in January 2006. Based on the Mills cross approach, previously unproven for riometry, the initial deployment was made using analog Butler matrices. To counteract for signal losses in the Butler matrices, preamplifiers were installed between the antennas and the Butler matrices. It was found that temperature variations in the hut caused gain changes in the preamplifiers and as they were outside of the calibration loop it was not possible to compensate for the changes. Various improvements took place including the replacement of the analog Butler matrices with the digital approach described previously. Operation as a full "production"'system, as described in this paper, began on March 5, 2007.

The higher spatial resolution afforded by ARIES has allowed a number of scientific discoveries which would not have been possible with existing $8 \times 8$ imaging riometers. For instance, Fig. 5 shows phase maps of pulsations at $3.2 \mathrm{mHz}$ for both IRIS and ARIES riometers for January 17, 2007 05:30 UT. The high $m$ number, 120, is close to the limiting spatial resolution for IRIS. The higher resolution of ARIES enables detection of much higher $m$ number events, up to 380 , some of which are not detectable with other imaging riometers. ${ }^{29}$

ARIES has also recorded the first direct measurements of absorption caused by ionospheric modification ("heating")
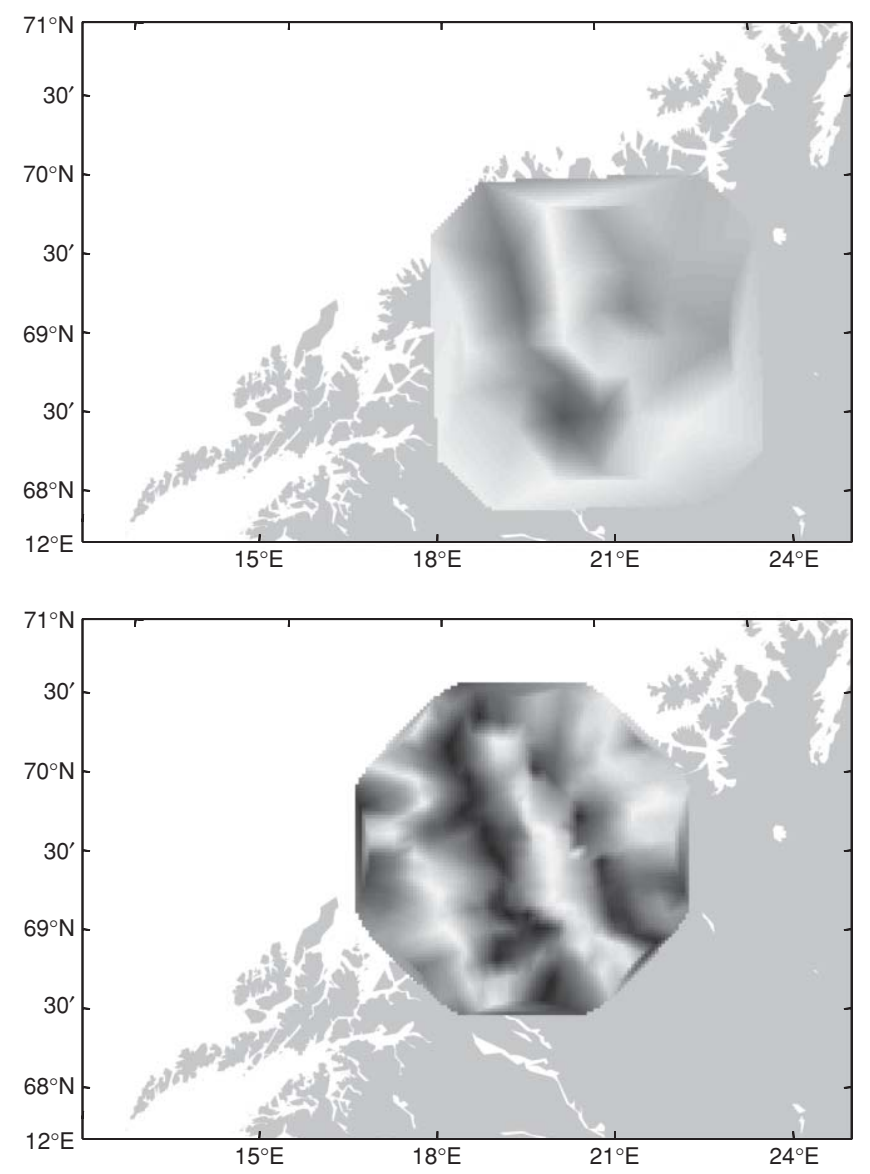

FIG. 5. Phase maps showing high $m$ number magnetospheric waves at $3.2 \mathrm{mHz}$ on January 17, 2007 05:30 UT. While the IRIS riometer (top panel) can observe the waves, the ARIES riometer (bottom panel) is capable of resolving much greater detail.

of the D-region by high-power HF radio waves. ${ }^{30}$ Due to the high-spatial resolution one of the ARIES beams is able to fit inside the heater beam. ARIES recorded an absorption value of $\approx 0.08 \mathrm{~dB}$, incomplete coverage (such as would occur with a riometer with poorer spatial resolution) would have reduced the measured absorption value, probably below the limit of the measurement precision. ARIES data have also been used in conjunction with data from the Kilpisjärvi IRIS riometer to measure the height of the absorbing layer and thus estimate the characteristic energies of the precipitating electrons. ${ }^{31}$ Similar studies have previously included optical data (e.g., Kosch et $a l .{ }^{32}$ ), limiting the procedure to cloud-free dark periods, whereas the riometer-only approach can be used without regard to weather or time of day.

ARIES ceased operation in August 2008 due to termination of its funding.

\section{FILLED ARRAY, WITH 8 × 8 ANTENNAS}

The modular design used in ARIES enables a filled array system with $8 \times 8=64$ antennas to be implemented using the same design with only two minor changes, the antenna layout and the FPGA program. While the spatial resolution is not better than the existing IRIS-type design the much higher integration time (952 ms compared to just $95 \mathrm{~ms}$ for 


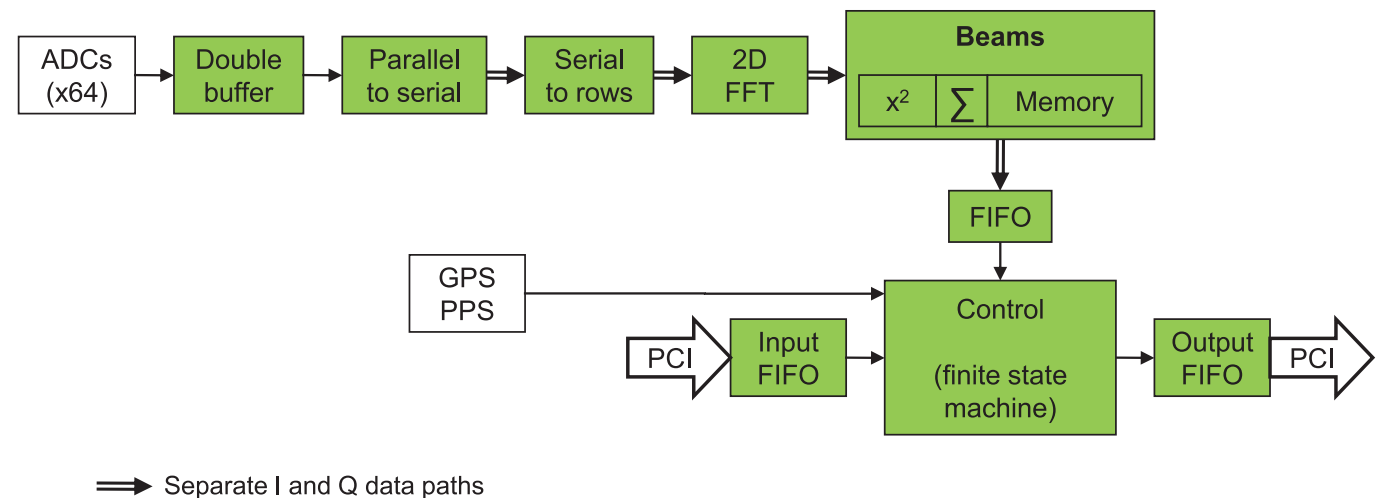

FIG. 6. (Color) Block diagram of the FPGA processing for the $8 \times 8$ filled array system. Filled boxes show stages processed within the FPGA. (FIFO denotes a first in, first out buffer.) Reproduced with permission from Imaging Riometer Operations and Data Processing Manual - Andøya, Norway. Copyright 2010 Lancaster University.

a $1 \mathrm{~s}$ cadence) results in a much improved measurement precision. Such a system was deployed at Andøya, Norway (Andøya IRIS, known as AIRIS) shortly after operation of ARIES commenced. The electronics hardware is identical to that used in ARIES, except for a few component changes in the receiver modules to reduce the receiver bandwidth; consequently, only the differences will be discussed below.

The antenna array comprises 64 crossed-dipoles, circularly polarized for $\mathrm{X}$-mode signals, arranged in an $8 \times 8$ grid with each antenna spaced $\lambda / 2$ from its neighbor. The sides of the antenna array are aligned with geographic North and East and the dipoles are aligned NE-SW and NW-SE. The antennas are of a different mechanical design to the ARIES antennas but otherwise are electrically identical. The bandwidthtime product for a filled array is much less critical than for a Mills cross system, so to reduce the possibility of interference the receiver bandwidth was reduced to $250 \mathrm{kHz}$. A block diagram of the FPGA processing is shown in Fig. 6. In comparison to the ARIES FPGA processing (Fig. 4), it can be seen that the dual linear Butler matrices (1D FFT) have been replaced by a single two-dimensional Butler matrix, implemented as a 2D FFT operation. The cross-correlator has been removed, which significantly decreases the computational requirements (see Table I). As a result the entire system can be implemented on a single Xilinx Virtex-II XC2V6000 fieldprogrammable gate array, which is a cheaper version of that used for ARIES, with a smaller number of programmable gates. The tapering and ADC offset removal functionality are not included as the FPGA program precedes those additions to the ARIES FPGA program. An almost identical system was later deployed at Ny-Ålesund, Svalbard in October 2008. As Ny-Ålesund is a radio-quiet location, where the probability of encountering interfering signals is much lower, the bandwidth of the system was enlarged to $1 \mathrm{MHz}$ to offset the smaller absorption values typically found inside the polar cap compared to those in auroral oval locations such as Kilpisjärvi and Andøya.

\section{FILLED ARRAY, 4 × 4 ANTENNAS}

The modular hardware design used in ARIES and AIRIS has permitted the development of a smaller $4 \times 4$ filled array imaging riometer system, with the advantages of lower cost and smaller footprint. The first system was deployed at the Indian Antarctic research station Maitri in February 2010. The design follows that of the $8 \times 8$ filled array system, albeit with a reduced number of antennas (16) and receivers (16). The standard Butler matrix design (16 beams, with no zenithal beam) was implemented as with fewer antennas; the corner beams do not suffer from grating lobes.

The block diagram of the FPGA processing, shown in Fig. 7, incorporates a number of software developments from the ARIES FPGA program. The 16 in-phase and 16 quadrature outputs from the ADCs are double-buffered and are then converted to one serial data stream. The signals are then passed through a first-order high-pass digital filter (as used in ARIES, $f_{c}=9.7 \mathrm{~Hz}$ ) to remove any offsets from the ADCs. The dc offset removal is applied independently to each of the 32 serial data values. Inside the gain control the signal level of each in-phase/quadrature pair is scaled with a 16 bit unsigned integer value. Each signal can be tapered and/or rotated by multiplying the in-phase/quadrature pair with a complex tapering coefficient (real and imaginary parts are each signed 16 bit values). If desired the dc offset removal, gain, and tapering stages can all be independently bypassed (not shown in the figure). The serial data stream is reordered to four serial data streams based on the antenna rows and a two-dimensional FFT computed. The signal values from each beam are squared and accumulated to provide a single power value for each beam.

In addition to the beam-formed output the FPGA also outputs power data from each receiver, which is equivalent to 16 wide-beam riometer outputs. For debugging purposes the input values for the power computation can be selected from one of several locations in the signal processing chain; before the dc offset removal stage, or after the dc offset removal, gain or tapering stages. A completely new feature is the inclusion of raw data recording, with a memory depth of 4094 samples recorded each second. This memory can be assigned to record from one receiver or split between two and operates in parallel to the normal beam-formed data. The raw data stream is saved from the same locations as the wide-beam data, but selected independently. 


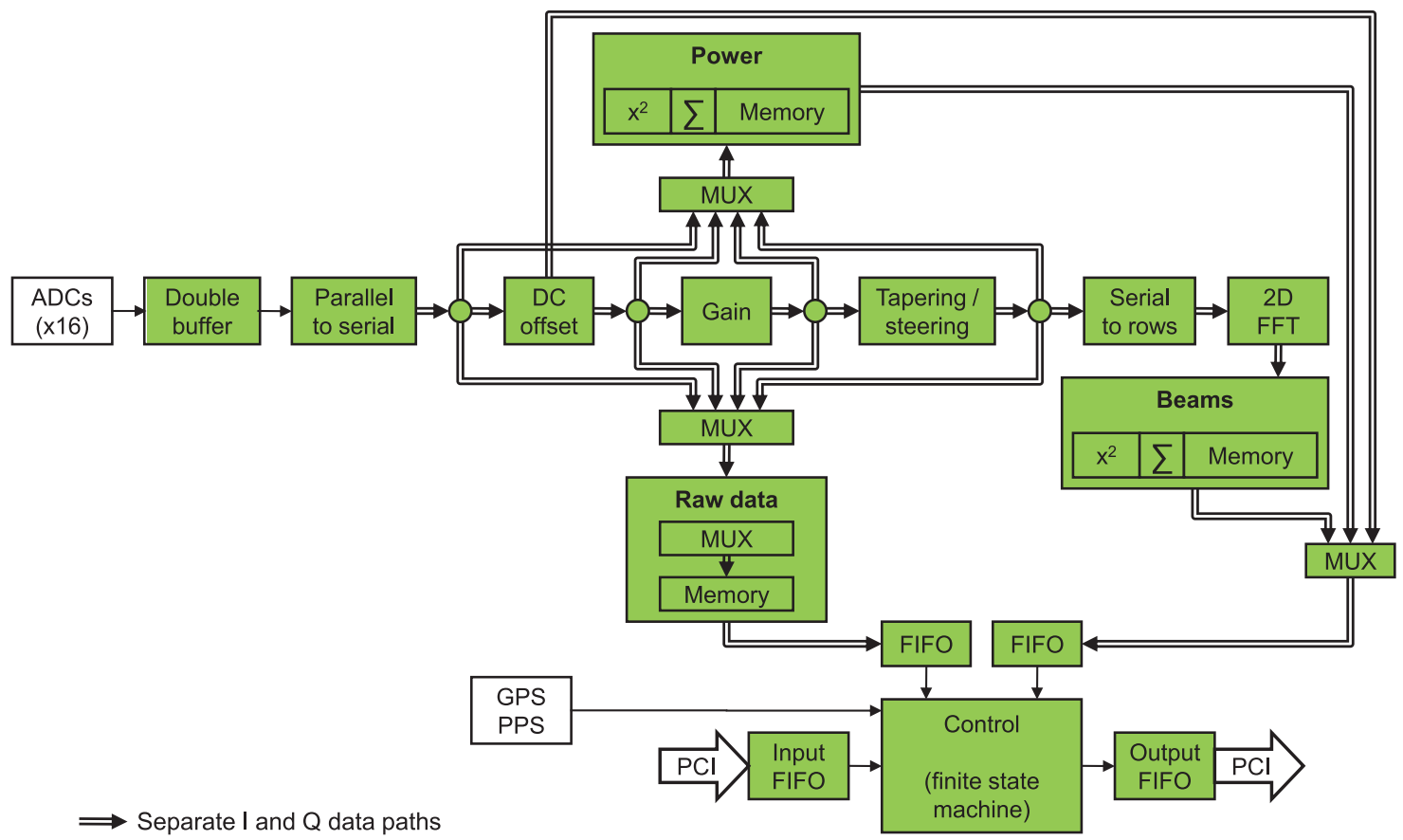

FIG. 7. (Color) Block diagram of the enhanced FPGA processing for the $4 \times 4$ filled array system. Filled boxes show stages processed within the FPGA. (FIFO denotes a first in, first out buffer.) Reproduced with permission from Imaging Riometer Operations and Data Processing Manual - Maitri, Antarctica. Copyright 2010 Lancaster University.

The FPGA functionality has now evolved to a level where no extra debugging programs are required, all necessary debugging and maintenance operations can be performed using the standard FPGA program, and in many cases without disturbing normal data recording. These refinements permit easier operation in remote locations; during installation of the system at Maitri, Antarctica it was discovered that one receiver module was not operating correctly, affecting data from two antennas. System testing carried out remotely from Lancaster, UK, indicated that the most likely fault was a local oscillator cable having worked loose during transit, which was confirmed when the back panel was removed. Hence, these refinements have proven extremely useful for fault recognition and remote operation.

\section{BEAM FORMING AND VALIDATION}

The beam projection for the ARIES Mills cross system is given in Fig. 8. Figures 9 and 10 show the beam projection from the $8 \times 8$ and $4 \times 4$ filled array systems, respectively. In all cases the field of view is approximately the same, 200 $\times 200 \mathrm{~km}$, and the beams are numbered row by row, starting from 1 in the NE corner.

The validity of the beam-forming process can be confirmed by comparing the received signal power from cosmic radio sources with the expected profile calculated using the theoretical antenna response. Provided that the beam-forming process is correct and the beam points in the correct direction, then the profiles of the measured and modeled radio source signal powers, including the timing of the maxima, will agree. Pointing errors which move the beam directly toward or away from the point where the radio source is closest to the beam center will not affect the timing of the maxima but the measured power level will not match the modeled value, other pointing errors will affect both the measured power level and the timing of the maxima.

For riometers located in the northern hemisphere the radio source Cassiopeia $\mathrm{A}$ was used and for Maitri in the

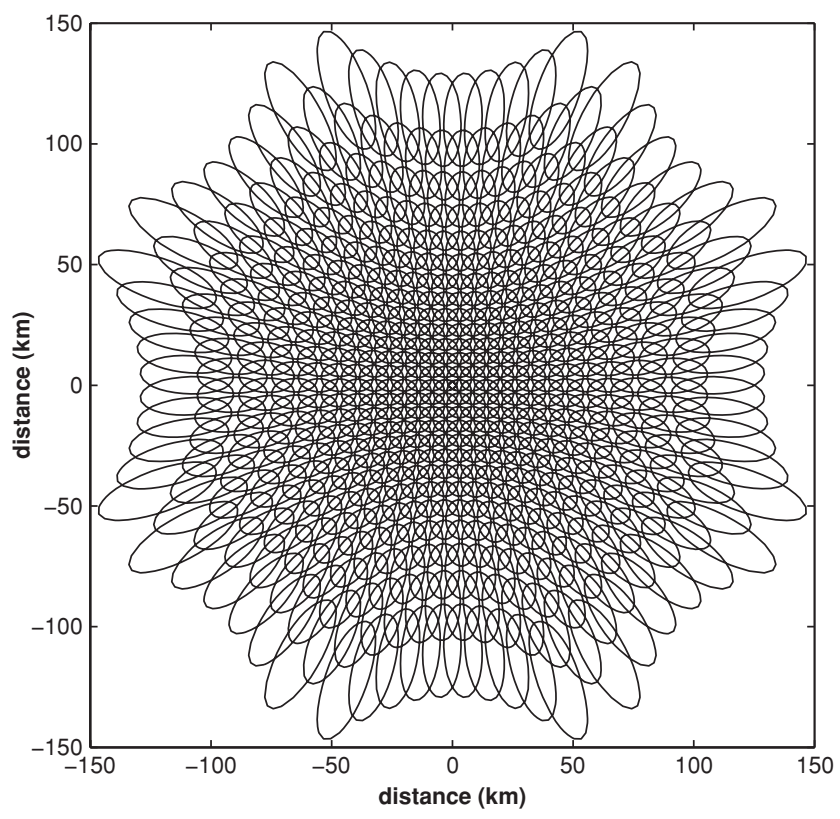

FIG. 8. Beam projection of the ARIES Mills cross. The $-3 \mathrm{~dB}$ beam contours are projected on the ionosphere at $90 \mathrm{~km}$ altitude. Only the 556 usable beams are shown, which are numbered left to right, row by row as: 108-117; 138$151 ; 169-184$; 200-217; 231-250; 262-283; 293-316; 325-348; 356-381; $388-413$; 420-445; 452-477; 484-509; 516-541; 548-573; 580-605; 612637; 644-669; 677-700; 709-732; 742-763; 775-794; 808-825; 841-856; 874-887; 908-917. 


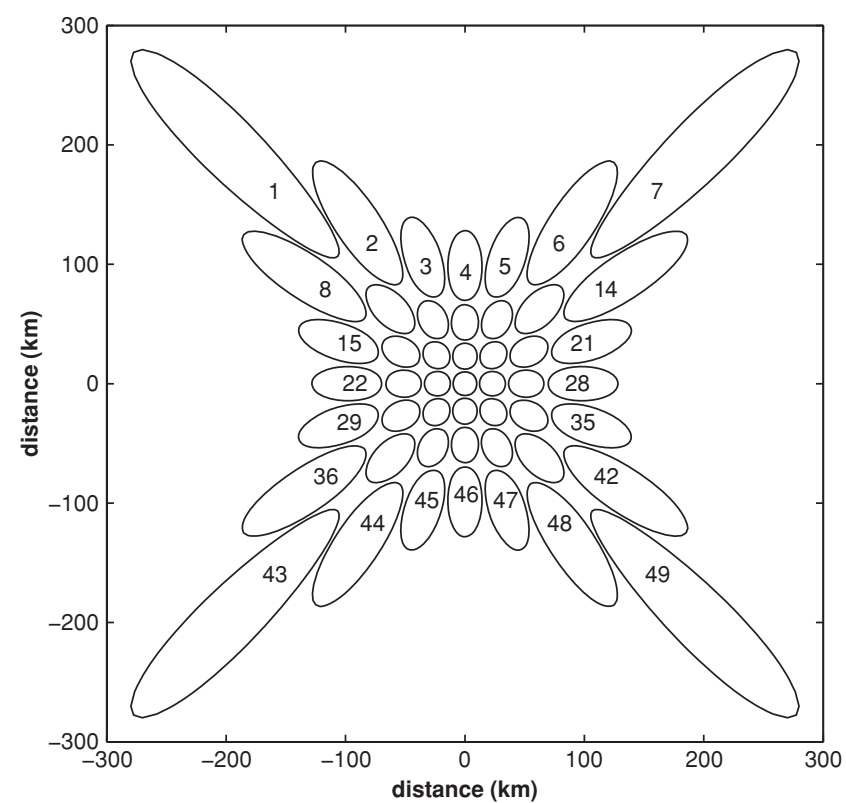

FIG. 9. Beam projection of the AIRIS $8 \times 8$ filled array. The $-3 \mathrm{~dB}$ beam contours are projected on the ionosphere at $90 \mathrm{~km}$ altitude. The beam numbers of the inner beams have been omitted for clarity.

southern hemisphere Sagittarius A* was used. For the beam widths in use they can be considered to be point sources. However, the recorded signal values contain contributions from the discrete radio source and from the diffuse cosmic noise background. Although all beams contain some contribution from the radio source we have only considered those beams where the locus of the radio source passes through the main lobe, as defined by the $-3 \mathrm{~dB}$ gain contour. For

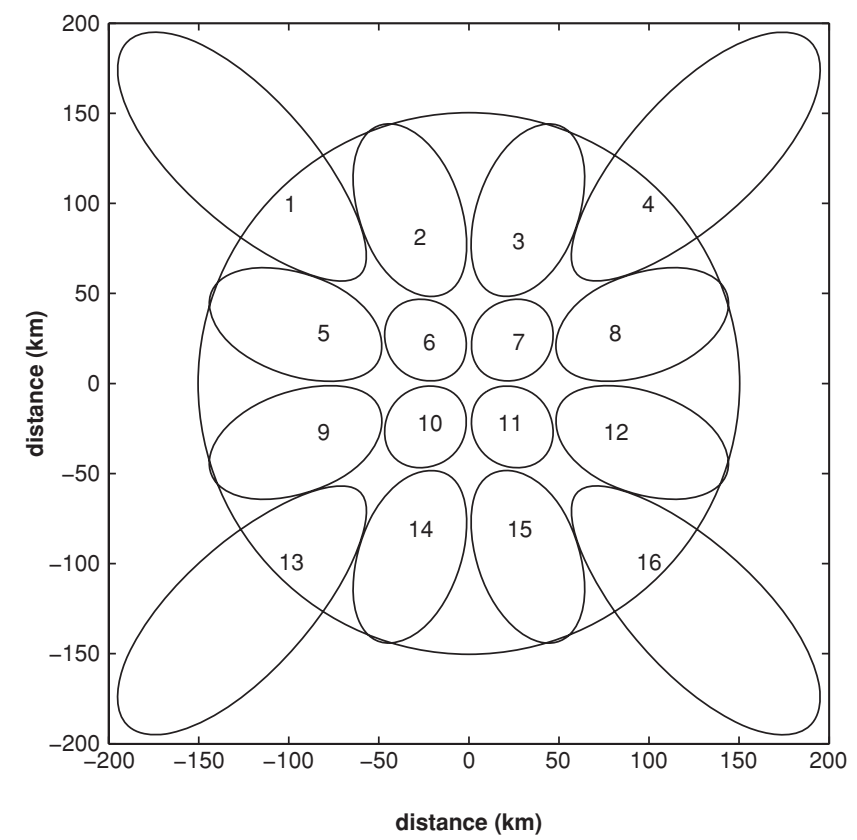

FIG. 10. Beam projection for the Maitri Indian Antarctic research station $4 \times 4$ filled array. The $-3 \mathrm{~dB}$ beam contours are projected on the ionosphere at $90 \mathrm{~km}$ altitude. The large circle shows the wide-beam riometer field of view. The 16 imaging beams are numbered. those beams we have estimated the radio source signal power by subtracting the background noise power obtained from a linear fit between the times when the radio source enters and leaves the $-20 \mathrm{~dB}$ gain contour of the main antenna lobe. The radio source signal power calculation assumes that the diffuse cosmic background (i.e., excluding the contribution from the radio source) varies linearly in the region surrounding the radio source; the validity of this assumption decreases as the beam width increases. To avoid problems arising from ionospheric scintillation the power values are taken from quiet day curves which were created using a method similar to that described by Browne, Hargreaves, and Honary. ${ }^{13}$

To confirm the validity of the method we present the results obtained for the IRIS system at Kilpisjärvi (Fig. 11), which uses analog Butler matrices. The figure shows 12 panels, one for each of the beams through which Cassiopeia A passes. The beam number is displayed in the lower left-hand corner of each panel. The $x$-axis represents time and on each panel the $x$-axis limits differ and are chosen to highlight the passage of the radio source through the beam. On the $y$-axis the radio source power is displayed (in decibels), relative to the power that would be received if the radio source were to pass through the center of the zenithal beam or the beam closest to the zenith for those systems without a zenithal beam. The $y$-axis scaling is identical for all panels ( -20 to $0 \mathrm{~dB}$ ). To account for the unknown system gain the recorded radio source signal powers have all been scaled by a common factor to provide the best fit against the modeled signal powers. From Fig. 11 it can be seen that there is good agreement between the measured and modeled signal powers and, in particular, with the times of the maxima. The minor discrepancies in signal powers we ascribe to errors in removing the cosmic background level. The analysis of the Kilpisjärvi IRIS system assumes an untapered system; in practice, a small degree of tapering exists due to unequal losses from the different antenna cable lengths. A similar beam-forming validation method is used by Senior $e t$ al. $^{30}$

Figure 12 shows the modeled and measured radio source signal powers for ARIES; from the excellent agreement, we deduce that the beam-forming process is working correctly. The results for AIRIS (Fig. 13) show good agreement, errors are of similar magnitude to the results seen for the Kilpisjärvi IRIS system. The results for the Maitri system, Fig. 14, show good agreement for beams $2,3,5$, and 8 . The results for beams 1 and 4 appear less convincing; however, the radio source passes close to the edge of these beams where the change in gain is most severe, so that small errors have a large effect on signal power ( $>1 \mathrm{~dB}$ per degree at the theoretical locations). It should also be remembered that the beams in the $4 \times 4$ system are larger and that the validity of the assumption of linearly varying cosmic noise background decreases as beam size increases. We believe these results show that the digital beam-forming process is working correctly for all system configurations.

\section{PERFORMANCE COMPARISONS}

Table I compares the specifications of seven different imaging riometer systems. The first three columns, IRIS, ${ }^{5}$ 

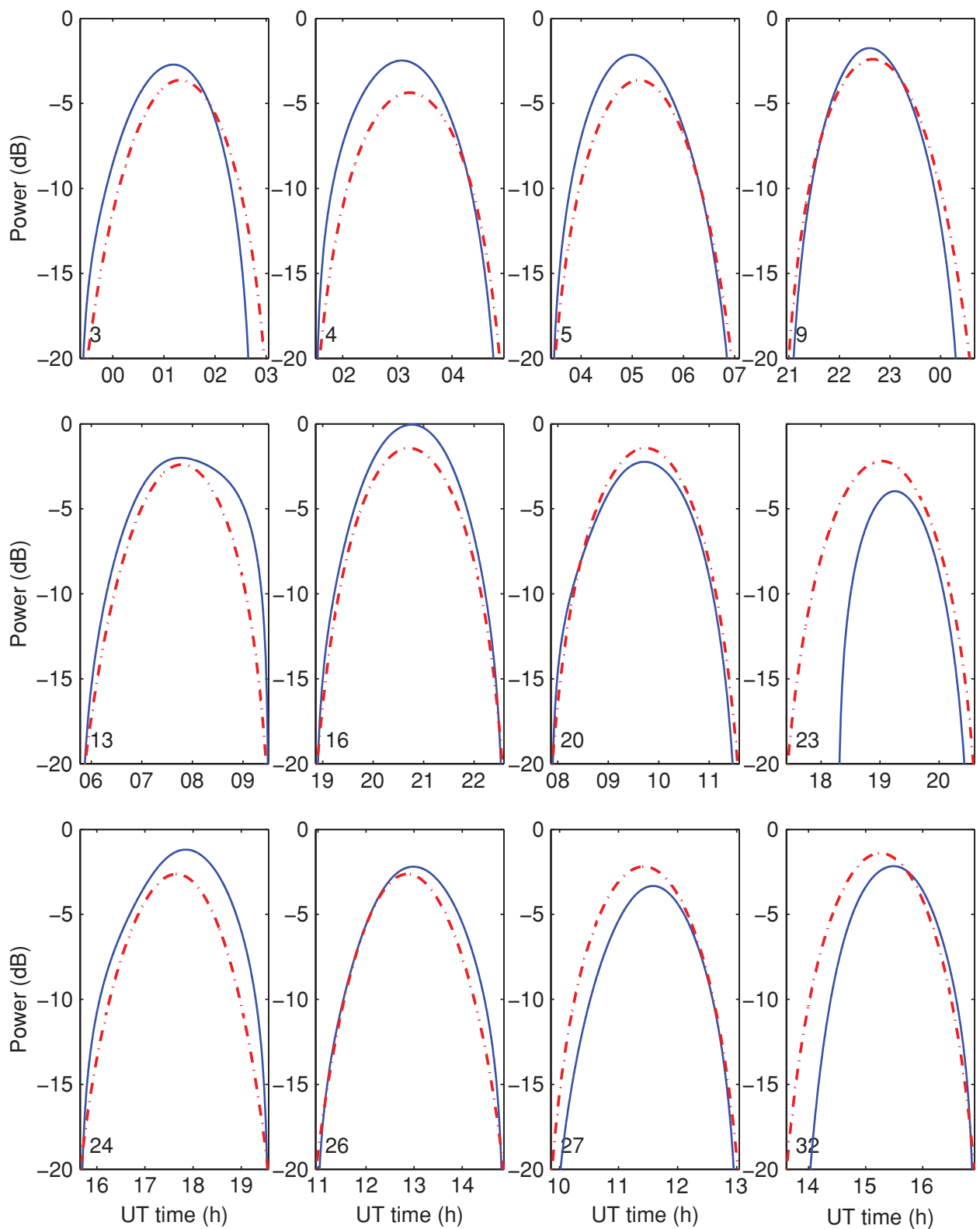

FIG. 11. (Color) Beam-forming check for the Kilpisjärvi IRIS system. Shown are the 12 beams through which Cassiopeia A passes. The contribution to the received power from Cassiopeia $\mathrm{A}$ is shown by solid lines, the modeled values are shown by dashed lines.

PENGUIN IRIS, ${ }^{9}$ and the Poker Flat imaging riometer, ${ }^{12}$ all use analog Butler matrices for beam forming. The remaining four columns describe the Mills cross system, the $8 \times 8$ filled array system (at both Andøya and $\mathrm{Ny}$ Ålesund) and $4 \times 4$ filled array system, all use digital beam forming.

The motivation for constructing a Mills cross riometer system was to provide higher spatial resolution than could be readily obtained with a filled array system. Compared to the Poker Flat imaging riometer ARIES has comparable spatial resolution (beam width) using significantly fewer antennas. The additional beams result in an oversampling of the imaging area, indicated by the beam separation $\left(3.53^{\circ}\right.$ at zenith) being much smaller than the beam width $\left(6.56^{\circ}\right.$ at zenith). The oversampling is demonstrated by the overlapping beams in the beam projection (Fig. 8).
An important specification for a riometer is its measurement precision. The precision, in decibels, is given by

$$
\mathrm{d} A=10 \log _{10}\left(1+\frac{1}{\mathrm{SNR}}\right) .
$$

For a filled array system the theoretical SNR is given by ${ }^{23}$

$$
\mathrm{SNR}_{\mathrm{FA}}=\sqrt{\tau B},
$$

where $\tau$ is the time constant (integration time) and $B$ the receiver bandwidth, assuming that the antenna power dominates over system noise. However, in many instruments a noisebalancing receiver is used, a device similar in design to the Dicke radiometer. ${ }^{33}$ The SNR is reduced by a factor of 2 because the antenna signal is being measured for only half of the time. This means that the relative accuracy of measuring either the antenna signal power or the noise source power will 

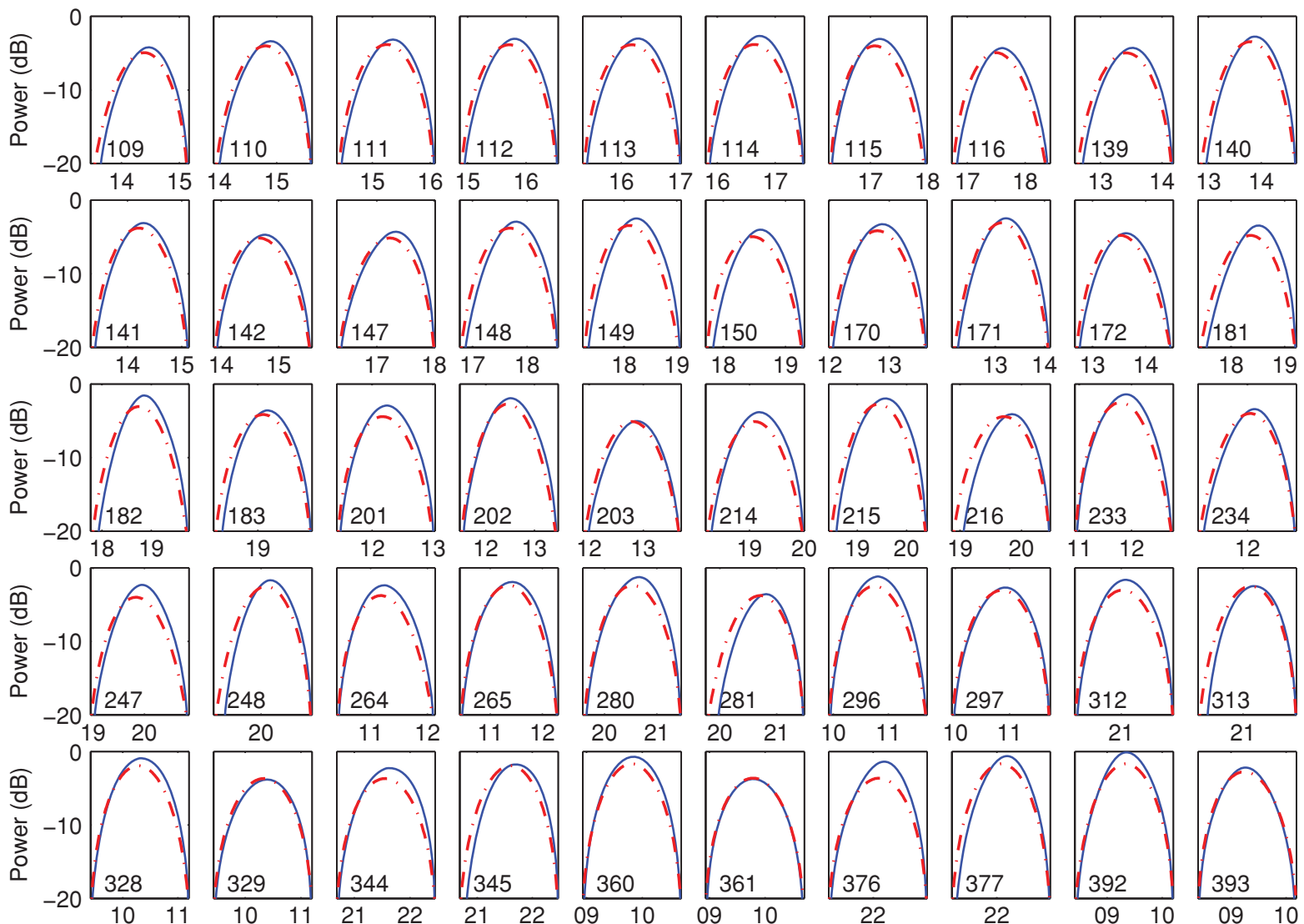

${ }_{10}{ }^{20} 11^{12}$
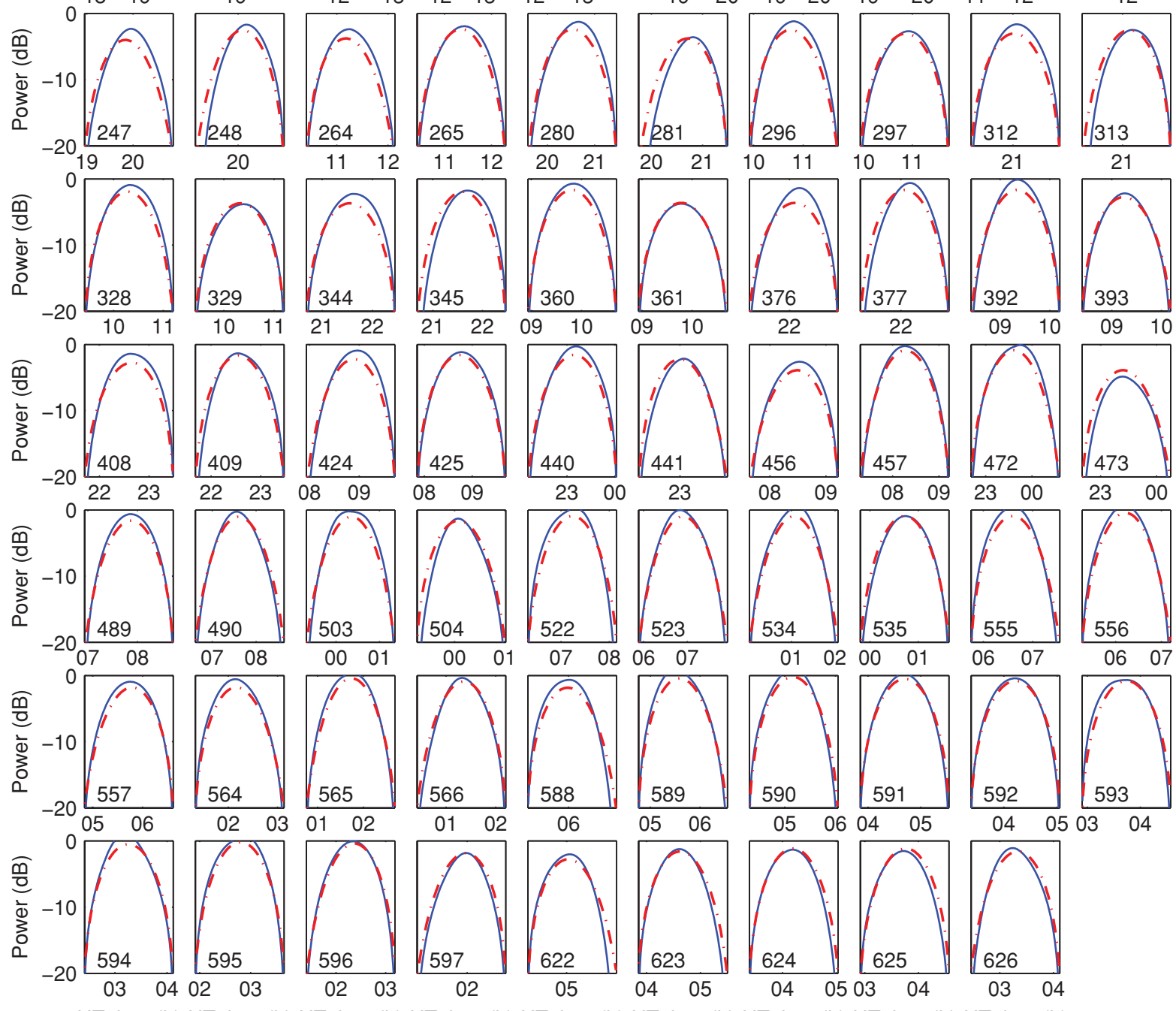

UT time (h) UT time (h) UT time (h) UT time (h) UT time (h) UT time (h) UT time (h) UT time (h) UT time (h)

FIG. 12. (Color) Beam-forming check for the ARIES system. Shown are the 89 beams through which Cassiopeia A passes. The contribution to the received power from Cassiopeia A is shown by solid lines, the modeled values are shown by dashed lines. (ARIES denotes Advanced Rio-Imaging Experiment in Scandinavia.)

be degraded by a factor of $\sqrt{2}$, and since the difference between two uncorrelated random variables is being taken, there is a further loss in accuracy of by a factor of $\sqrt{2}$. Therefore, the SNR is reduced by a factor of $2 .{ }^{34}$ From this it follows that the SNR of a filled-array imaging riometer employing a noise-balancing receiver is given by the equation

$$
\mathrm{SNR}_{\mathrm{NB}}=\frac{\sqrt{\tau B}}{2} .
$$



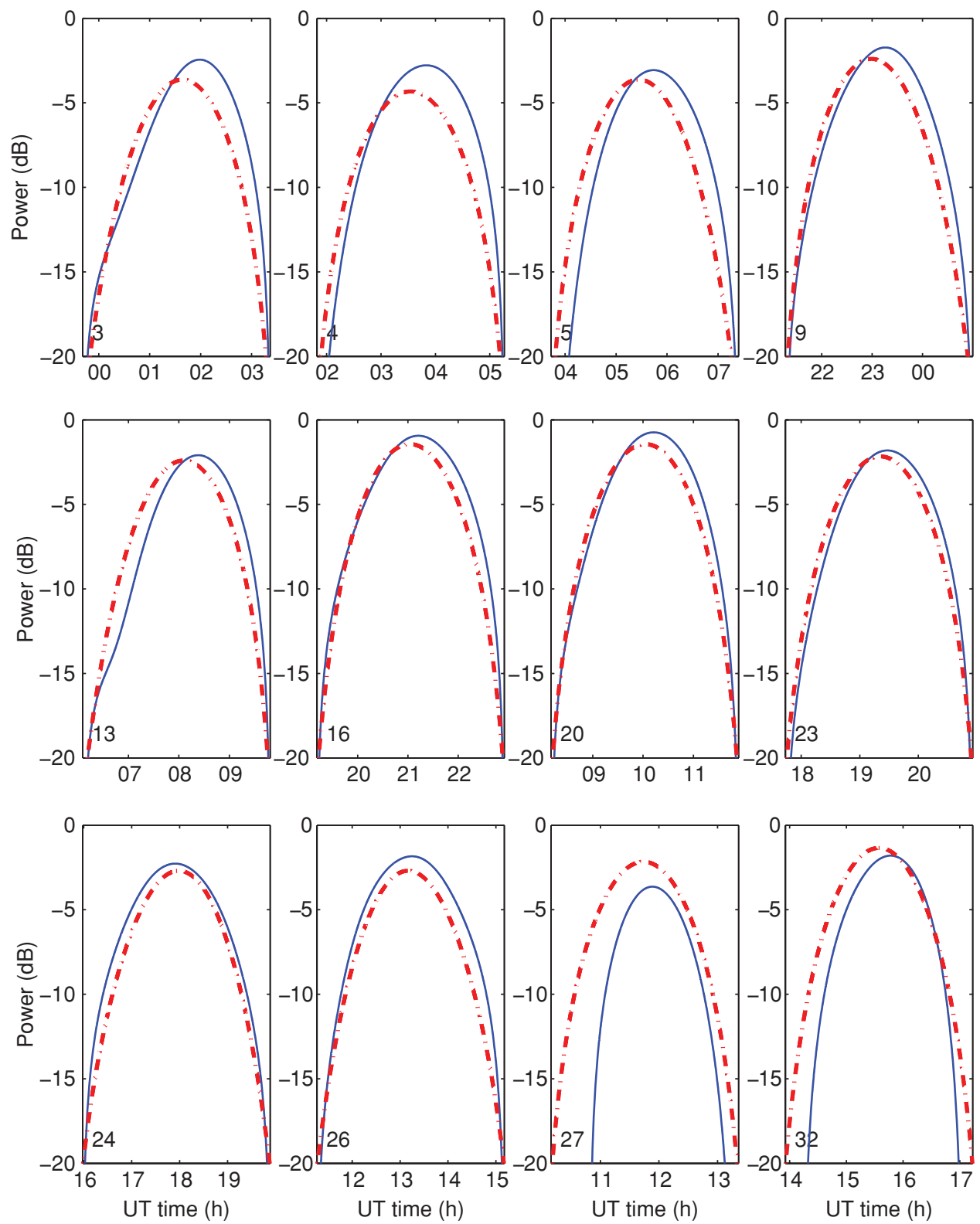

FIG. 13. (Color) Beam-forming check for the AIRIS system. Shown are the 12 beams through which Cassiopeia A passes. The contribution to the received power from Cassiopeia A is shown by solid lines, the modeled values are shown by dashed lines. (AIRIS denotes Andøya Imaging Riometer for Ionospheric Studies.)

The signal-to-noise ratio has been measured from five different riometers using the same method; for each beam the ratio of the mean to standard deviation (using detrended data) in a 15 min sliding window over an entire day was computed and the lowest value recorded in order to eliminate any possible adverse effects from scintillation. The median of the per-beam SNR values is then used as the system signal-tonoise ratio. The results are given in Table I. As validation of the SNR measurements it can be seen that the measured precision of the IRIS riometer system at Kilpisjärvi is only slightly less than that can be achieved theoretically.

Theoretical calculations of the SNR performance of a Mills cross system by Hagfors, Grill, and Honary ${ }^{24}$ suggested that the time-bandwidth product must be increased by a factor of 100-900 compared to a filled array for similar performance to be achieved. The bandwidth-time product for
ARIES is just over 50 times greater than that for IRIS; however, the absence of noise balancing in ARIES results in an effective increase of just over 100 times. Thus the measurement precision of ARIES is predicted to be comparable to that of IRIS.

Nielsen, Honary, and Grill ${ }^{23}$ gave an equation for the expected signal-to-noise ratio for a Mills cross system. Due to an error in the working the equation given is incorrect; the SNR is actually given by

$$
\mathrm{SNR}_{\mathrm{MC}}=\frac{\sqrt{\tau B}}{N}
$$

where $N$ is the number of subdivisions in a fan beam. For the untapered case $N=32$, for the tapered case $N \approx$ $32 \times\left(4.5^{\circ} / 6.56^{\circ}\right)$. This corrected SNR calculation suggests that the theoretical measurement precision is $0.097 \mathrm{~dB}$. The 

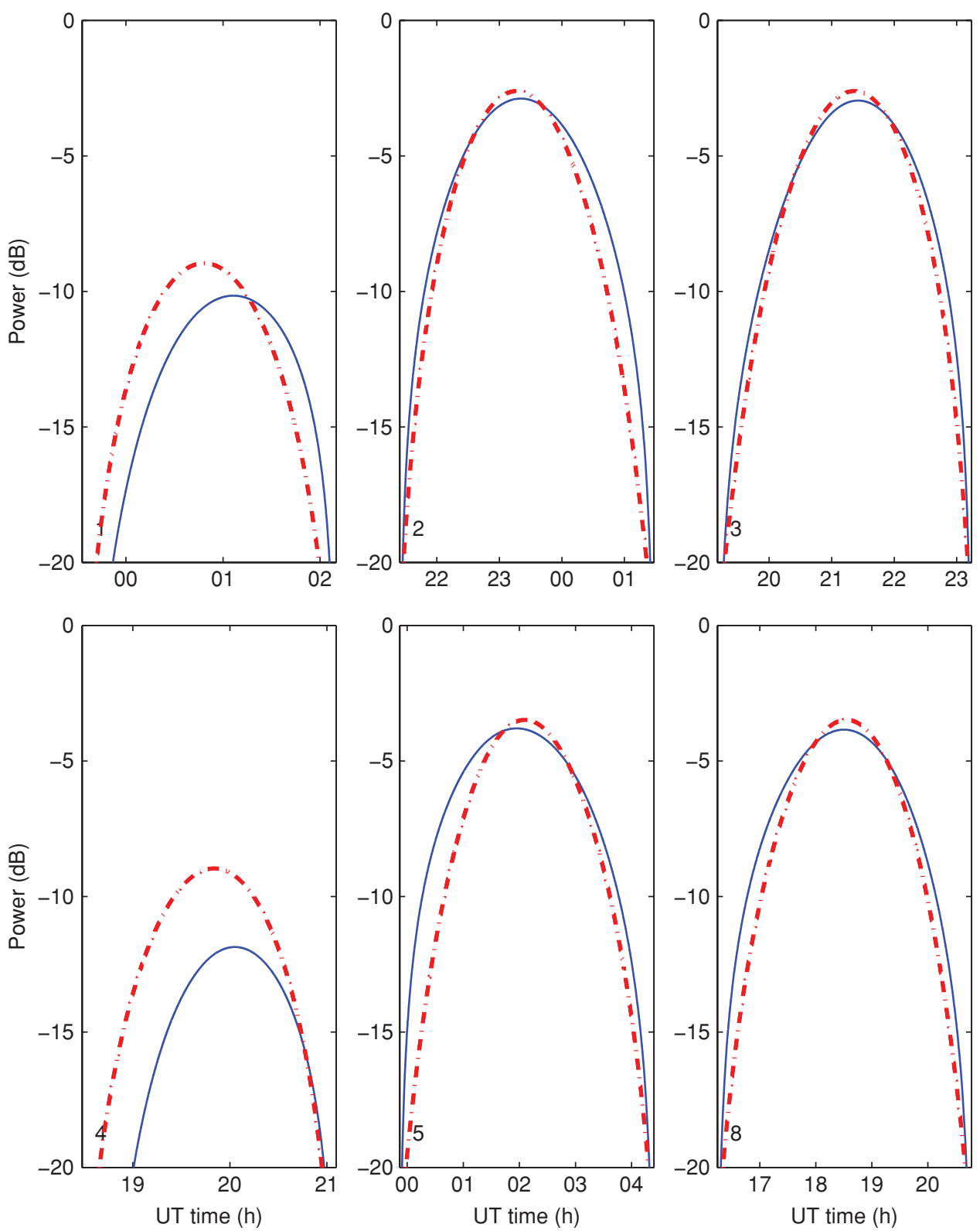

FIG. 14. (Color) Beam-forming check for Maitri system. Shown are the six beams through which Sagittarius A* passes. The contribution to the received power from Sagittarius A* is shown by solid lines, the modeled values are shown by dashed lines.

measured value for ARIES is better than this, $0.045 \mathrm{~dB}$. This is possible because the assumptions of even cosmic background and equal antenna gain for each subdivision are not correct; certain combinations of fan beams and sidereal times can result in a stronger signal appearing in the intersecting region compared to the nonintersecting parts of the fan beams, resulting in a higher SNR than predicted.

The motivation for creating a filled phased array system with digital beam forming was to improve the measurement precision compared to the existing riometers. Table I shows that for AIRIS the theoretical precision, $0.0089 \mathrm{~dB}$, is nearly five times better than any other system operating with a $1 \mathrm{~s}$ cadence and also better than the PENGUIN IRIS system operating at $12 \mathrm{~s}$ cadence. Comparable noise performance from an IRIS system would require that the data be postintegrated to $25 \mathrm{~s}$ cadence. The performance increase arises in part from the enlarged bandwidth ( $25 \%$ increase), the absence of noise balancing, but mostly from the much larger integration time $(10 \times$ increase). With its wider $1 \mathrm{MHz}$ bandwidth the similar system at Ny-Ålesund performs even better. The $4 \times 4=16$ filled array system at Maitri offers identical noise performance to AIRIS. Although it features fewer beams, its smaller footprint enables deployment to sites which lack the space requirements for an $8 \times 8=64$ antenna array, such as that found at Maitri.

Included in Table I is a calculation of how much data are potentially affected by scintillation, as determined by the loci of the radio sources Cassiopeia A, Cygnus A, and Sagittarius $\mathrm{A}^{*}$ intersecting with the $-3 \mathrm{~dB}$ gain contour of the beams. The susceptibility is largely determined by the instrument's latitude; however, the riometer design is also a factor. Typically, only one beam at any time is affected by a given radio source; hence, riometers with a larger number of beams appear less affected. However, the narrower beam widths of 
such systems mean that scintillation is likely to be stronger at the times when it does occur, as less comic background signal is received.

\section{SUMMARY}

A new class of imaging riometers, using digital beam forming, has been implemented; with modular hardware the design can be easily optimized for improved spatial resolution or measurement precision. We have shown, for the first time, that a riometer system based upon the Mills cross is a viable alternative to a filled phased array, giving improved spatial resolution and comparable measurement precision for the same cadence and number of antennas when compared to existing analog imaging riometers. The higher spatial resolution achieved by ARIES has resulted in a number of important scientific discoveries, as described in Sec. II D.

We have also shown that a filled phased array system utilizing digital beam forming with independent receivers for each antenna can provide much better measurement precision compared to existing imaging riometers using analog Butler matrices. Improved measurement precision is useful for various scientific studies, e.g., in order to measure the solarcontrolled absorption.

The digital implementation allows a number of improvements to be easily incorporated. For system testing and diagnosis the FPGA can be reconfigured so that outputs from each antenna/receiver can be viewed either as integrated $1 \mathrm{~s}$ data or as raw data samples. As physical access is not required this simplifies operation in harsh and remote environments such as the Arctic and Antarctic. In the latest software version, as deployed to Maitri, this functionality is part of the standard FPGA program so that some testing is possible without disturbing normal data recording, as well as providing widebeam riometer data.

Further improvements with a digital beam-forming approach are possible. For instance, it is possible to implement both modified and nonmodified Butler matrix designs simultaneously in order to obtain overlapping beam radiation patterns for higher spatial resolution. Alternatively, by computing and recording the cross-products any beam arrangement could be obtained by postprocessing of the data.

\section{ACKNOWLEDGMENTS}

We are deeply indebted to the late Tor Hagfors, who provided invaluable advice and input, especially during his threemonth visit to Lancaster University made possible through a Royal Society Visiting Fellowship. We gratefully acknowledge the support given by Andrew Senior. We thank Paul Wild for his assistance calculating the side lobe levels for the ARIES and IRIS riometers, and Mathew Beharrell for the riometer phase maps.

ARIES development was funded by the UK's Particle Physics and Astronomy Research Council (PPARC), now the Science and Technology Facilities Council (STFC). We would like to thank all at the European Incoherent Scatter Radar (EISCAT) facility in Troms $\varnothing$ for their practical assistance with the installation and operation of ARIES. We thank our colleagues at the Arctic Lidar Observatory for Middle Atmosphere Research (ALOMAR), Andøya, Norway, for their support deploying and operating AIRIS; our colleagues at the Polar Research Institute of China for their support in deploying the riometer at Ny-Ålesund, Norway; and also our colleagues at the Indian Institute of Geomagnetism in Mumbai for deploying the riometer at Maitri, Antarctica.

${ }^{1}$ C. G. Little and H. Leinbach, Proc. IRE 47, 315 (1959).

${ }^{2}$ E. Nielsen and W. I. Axford, Nature (London) 267, 502 (1977).

${ }^{3}$ T. Kikuchi, H. Yamagishi, and N. Sato, Geophys. Res. Lett. 15, 168 (1988).

${ }^{4}$ H. Yamagishi and T. Kikuchi, Antarct. Rec. 33, 17 (1989).

${ }^{5}$ D. L. Detrick and T. J. Rosenberg, Radio Sci. 25, 325 (1990).

${ }^{6}$ R. J. Mailloux, Phased Array Antenna Handbook, 2nd ed. (Artech House, Boston, 2005)

${ }^{7}$ P. J. Muenzer, Nachrichtentechnische Z. 25, 419 (1972).

${ }^{8}$ J. Butler and R. Lowe, Electron. Des. 12, 170 (1961).

${ }^{9}$ D. L. Detrick and L. Lutz, Antarct. J. U.S. 29, 354 (1994).

${ }^{10}$ See http://www.spase-group.org/data/doc/spase-2_2_0.pdf for Space Physics Archive Search and Extract (SPASE) data dictionary.

${ }^{11}$ H. Yamagishi, M. Nishino, M. Sato, Y. Kato, M. Kojima, N. Sato, and T. Kikuchi, Antarct. Rec. 36, 227 (1992).

${ }^{12}$ Y. Murayama, H. Mori, S. Kainuma, M. Ishi, I. Nishimuta, K. Igarashi, H. Yamagishi, and M. Nishino, J. Atmos. Sol.-Terr. Phys. 59, 925 (1997).

${ }^{13}$ S. Browne, J. K. Hargreaves, and B. Honary, Electron. Commun. Eng. J. 7, 209 (1995).

${ }^{14}$ A. Senior, M. J. Kosch, and F. Honary, Ann. Geophys. 26, 3831 (2008).

${ }^{15}$ M. J. Jarvis, R. E. Hibbins, M. J. Taylor, and T. J. Rosenberg, Geophys. Res. Lett. 30, 2/1 (2003).

${ }^{16}$ M. Beharrell and F. Honary, J. Geophys. Res., [Space Phys.] 113, 1 (2008).

${ }^{17}$ M. Harrich, M. Friedrich, S. R. Marple, and K. M. Torkar, Adv. Radio Sci. - Kleinheubacher Ber. 1, 325 (2003).

${ }^{18}$ E. Nielsen and T. Hagfors, . Atmos. Sol.-Terr. Phys. 59, 939 (1997).

${ }^{19}$ B. Y. Mills and A. G. Little, Aust. J. Phys. 6, 272 (1953).

${ }^{20}$ E. Nielsen, "Antenna system for a high resolution imaging riometer," Tech. Rep. MPAE-W-03-01-04 (Max-Planck-Institut für Aeronomie, Lindau, Germany, 2001).

${ }^{21}$ B. Y. Mills, Aust. J. Sci. Res., Ser. A 5, 266 (1952).

${ }^{22}$ W. N. Christiansen and D. S. Mathewson, Proc. IRE 46, 127 (1958).

${ }^{23}$ E. Nielsen, F. Honary, and M. Grill, Ann. Geophys. 22, 1687 (2004).

${ }^{24}$ T. Hagfors, M. Grill, and F. Honary, Radio Sci. 38, 17/1 (2003).

${ }^{25}$ G. H. Brown, Electronics 9, 15 (1936).

${ }^{26} \mathrm{M}$. Grill, K. Barratt, and F. Honary, Earth Sci. Inform. 3(1-2), 111-117 (2010).

${ }^{27}$ S. R. Marple and F. Honary, Adv. Polar Upper Atmos. Res. 18, 120 (2004).

${ }^{28}$ M. Grill, "Technological advances in imaging riometry," Ph.D. dissertation (University of Lancaster, UK, 2007).

${ }^{29}$ M. Beharrell, A. J. Kavanagh, and F. Honary, J. Geophys. Res., [Space Phys.] 115, 1 (2010).

${ }^{30}$ A. Senior, M. T. Rietveld, F. Honary, W. Singer, and M. J. Kosch, "Measurements and modeling of cosmic noise absorption changes due to radio heating of the D region ionosphere," J. Geophys. Res., [Space Phys.](in press).

${ }^{31}$ P. Wild, F. Honary, A. J. Kavanagh, and A. Senior, J. Geophys. Res., [Space Phys.] 115, 1 (2010).

${ }^{32}$ M. J. Kosch, F. Honary, C. F. del Pozo, S. R. Marple, and T. Hagfors, J. Geophys. Res., [Space Phys.] 106, 28925 (2001).

${ }^{33}$ R. H. Dicke, Rev. Sci. Instrum. 17, 268 (1946).

${ }^{34}$ B. F. Burke and F. Graham-Smith, An Introduction to Radio Astronomy, 2nd ed. (Cambridge University Press, Cambridge, 1997). 